\title{
Sozioökonomische Bildung als ein zentrales Paradigma für den Lehrplan "Geographie und Wirtschaftliche Bildung" 2020 der Sekundarstufe I
}

*christian.fridrich@phwien.ac.at, Pädagogische Hochschule Wien

eingereicht am: 05.11.2019, akzeptiert am: 17.02.2020

Bereits mit den GW-Lehrplänen 1985 und 2000 wurde eine sozialwissenschaftliche Orientierung des Faches eingeleitet, die mit der aktuellen Lehrplangestaltung unter Integration von Ansätzen und Prinzipien der sozioökonomischen Bildung fortgeführt, verfeinert und vertieft wurde. Es wird den Fragen nachgegangen, in welcher Weise und für welche Bereiche Konzepte und Ergebnisse des aktuellen Diskurses der sozioökonomischen Bildung sowie diesbezügliche empirische Befunde in die Gestaltung des neuen Lehrplans für das Fach „Geographie und Wirtschaftliche Bildung“ integriert wurden. Aus differenzierten Analysen wird erkennbar, dass diese Integration systematisch, umfassend, für alle Bereiche des Lehrplans und bezüglich aller Kompetenzformulierungen erfolgte.

Keywords: Lehrplan, Geographie und Wirtschaftliche Bildung, Wirtschaftskunde, sozioökonomische Bildung

\section{Socio-economic education as a central paradigm for the "Geography and Economics" curriculum of 2020 for lower secondary}

With the curricula of "Geography and Economics" 1985 and 2000, a socio-scientific orientation of the subject was already introduced, which was continued, refined and deepened with the current curriculum design, integrating approaches and principles of socio-economic education. This paper examines the ways and areas in which concepts and outcomes of the current discourse on socio-economic education, as well as related empirical findings, have been integrated into the design of the new curriculum for geography and economics education. Differentiated analyses show that this integration was systematic and comprehensive, targeting all areas of the curriculum and all competence descriptors.

Keywords: curriculum, Geography and Economics Education, economics, socio-economic education

\section{Annäherung}

Im Hinblick auf den Titel dieses Beitrags sind vorab zwei Aspekte zu klären. Erstens liegt die Betonung bezüglich der sozioökonomischen Bildung auf „ein zentrales Paradigma für den GW-Lehrplan 2020“1. Daneben existieren zahlreiche andere wichtige Gestaltungsfaktoren, die bei der Neuerstellung des GWLehrplans zum Tragen kamen, nämlich beispielsweise

\footnotetext{
Wenn hier verkürzt immer wieder vom Lehrplan 2020 gesprochen wird, handelt es sich um einen weit fortgeschrittenen, bereits intensiv diskutierten und überarbeiteten Entwurf der ministeriellen Lehrplanarbeitsgruppe für GW (siehe in GW-Unterricht 156 eine kurze Erläuterung zum Stand der Arbeit bei Fridrich \& Koller 2019 sowie den Entwurfstext bei Chreiska-Höbinger et al. 2019).
}

neue fachdidaktische und allgemeindidaktische Entwicklungen und Erkenntnisse, wie etwa Kompetenzorientierung, außerdem Intentionen und Konzepte der Bildung für nachhaltige Entwicklung sowie der politischen Bildung, aktuelle sozial- und physiogeographische Themen, die zielgerichtete Integration von Geomedien in den Unterricht etc. ${ }^{2}$ Dies alles fundiert in einem einzigen Beitrag darstellen zu wollen, würde

2 Alle eben angeführten Aspekte und Themenbereiche - einschließlich der sozioökonomischen Bildung - wurden von Mitgliedern der Lehrplangruppe in vernetzter Weise eingebracht. Die Kerngruppe besteht - alphabethisch gereiht - aus: Carina Chreiska-Höbinger, Christian Fridrich, Stefan Hinsch, Paul Hofmann, Herbert Pichler und Marcel Vorage; die Erweiterungsgruppe aus: Thomas Jekel, Lars Keller und Alfons Koller. 
den Rahmen bei weitem sprengen, weswegen hier auf die Relevanz der sozioökonomischen Bildung, die mit den anderen Themenbereichen eng verflochten ist, für die Lehrplangestaltung fokussiert wird.

Zweitens ist nachzufragen, inwiefern bereits der „alte“ Lehrplan 2000, der wesentlich auf dem GWLehrplan 1985 basiert, Elemente des Paradigmas der sozioökonomischen Bildung integriert, ohne freilich diese Bezeichnung explizit anzuführen. Das Paradigma der sozioökonomischen Bildung ist durch folgende Prinzipien gekennzeichnet, die altersgemäß im Unterricht umzusetzen sind (Hedtke 2019: 95-235; siehe vergleichbar auch die „Grundgedanken“ der sozioökonomischen Bildung bei Famulla 2019: 25-29):

1. Subjektorientierung: Dieses Prinzip lässt sich in den zwei eng miteinander verbundenen Dimensionen Erfahrungsorientierung und Lebensweltorientierung verorten. Einerseits stehen Lernende mit ihren Erfahrungen, Erlebnissen, Alltagsvorstellungen und Weltorientierungen im Zentrum des Unterrichts. Andererseits bedeutet die daraus resultierende Lebensweltorientierung, Herausforderungen aus dieser aufzugreifen, kritisch zu reflektieren und junge Menschen in unterschiedlichen gesellschaftlichen Kontexten handlungsfähig zu machen (Hedtke 2019: 104-160; Famulla 2019: 25f.; siehe dazu auch die schüler- und handlungsorientierte Perspektive bei Engartner \& Krisanthan 2014b: 166 sowie die kritisierte Durchsetzung von Interessen Dritter bei Engartner 2019c; oder in Bezug auf Lernprozesse die Schüler- und Lebensweltorientierung sowie die Kompetenz- und Handlungsorientierung bei Fridrich \& Hofmann-Schneller 2017: 56).

2. Problemorientierung: Bezüglich des Gegenstandsbereiches wird Problembezug bedeutsam, indem besonders relevante Herausforderungen und Probleme bildungsrelevant werden. Diese lassen sich in den drei großen Bereichen Haushalt, Arbeitswelt und Gesellschaft verorten, in denen es eine Vielzahl und Vielfalt an - teilweise einander widersprechenden - Handlungsoptionen gibt (Hedtke 2019: 161-175; Famulla 2019: 28; siehe auch die problemorientierte Methodologie und die Handlungsbereiche bei Engartner \& Krisanthan 2014b: 166-170; oder bezüglich der Gegenstandsbereiche den Aktualitäts- und Zukunftsbezug bei Fridrich \& Hofmann-Schneller 2017: 56).

3. Sozialwissenschaftlichkeit: Wenn Wirtschaft als gesellschaftlich eingebettet und konstituiert verstanden wird, liegt es auf der Hand, Wirtschaft multidisziplinär sowie mit Zugängen, Theorien und Methoden der Sozialwissenschaften zu erschließen. Viele Konzepte wie Arbeit, Leistung, Konsum, Interesse, Staat, Institution, Organisati- on etc. können adäquat nur transdisziplinär sowie wirtschafts- und sozialwissenschaftlich erschlossen werden. Wirtschaft ist somit immer in einem Wirkungsgefüge mit den weiteren Komponenten Gesellschaft, Politik und Umwelt eingewoben (Hedtke 2019: 176-198; Famulla 2019: 26-28; siehe auch die kontextualisierte Perspektive und die Handlungsbereiche bei Engartner \& Krisanthan 2014b: 166-170; oder in Bezug auf Wissenschaft die ausgeführte Wissenschaftsorientierung bei Fridrich \& Hofmann-Schneller 2017: 56).

4. Pluralität: Das Prinzip der Pluralität ist mit der inhaltlichen Mehrperspektivität und der Kontroversität eng verbunden. Pluralismus ist ein essenzielles Merkmal moderner Gesellschaften, weswegen im Unterricht auch unterschiedliche Leitbilder und Praktiken des Wirtschaftens in der Gesellschaft bearbeitet werden sollen. Dies unter Einbeziehung verschiedener wirtschaftlicher Paradigmen sowie Welt- und Menschenbilder (Hedtke 2019: 199-235; Famulla 2019: 28-29; siehe dazu auch die wertoffenen und pluralistischen „Einstellungen" bei Engartner \& Krisanthan 2014b: 166; oder in Bezug auf Wissenschaft sowohl inhaltliche Mehrperspektivität und Pluralismus bei Fridrich \& Hofmann-Schneller 2017: 56).

Schon bei einer ersten Analyse des Lehrplans 2000 für die Sekundarstufe I fällt auf, dass der in räumlichen und wirtschaftlichen Zusammenhängen handelnde Mensch im Mittelpunkt der Betrachtungen steht und dass räumliche, gesellschaftliche und wirtschaftliche Aspekte vernetzt behandelt werden sollen, womit zunächst zwei von vier Grundgedanken der sozioökonomischen Bildung (Famulla 2019: 25-29) angesprochen werden. So zielen 13 der 17 Themenkreise auf eine integrierte Bearbeitung von geographisch-wirtschaftskundlichen Inhalten ab. Explizit wird diese Vernetzung in den didaktischen Grundsätzen gefordert: „Geographische und wirtschaftskundliche Inhalte sollen im Unterricht nicht nebeneinander stehend getrennt, sondern in starkem Maße miteinander verflochten in vergleichender Darstellung aller Kontinente unter möglichst häufiger Berücksichtigung Österreichs behandelt werden" (Bundeskanzleramt 2019: 75). Lediglich zwei Themenkreise beziehen sich auf fast ausschließlich geographische Aspekte, nämlich „Ein Blick auf die Erde“ (5. Schulstufe) und „Lebensraum Österreich“ (7. Schulstufe), während nur zwei andere Themenkreise weitestgehend auf wirtschaftliche Schwerpunkte fokussieren, es sind dies „Wirtschaften im privaten Haushalt" (7. Schulstufe) und „Volkswirtschaftliche Zusammenhänge: Österreich - Europa" (7. Schulstufe). Der Mensch im Mittelpunkt des GW-Unterrichts kann noch in einer zweiten Weise verstanden werden, wenn nämlich Schülerinnen 
und Schüler im Zentrum des Unterrichtsgeschehens stehen und dann beispielsweise von ihren Erfahrungen, Vorkenntnissen, Alltagsvorstellungen sowie subjektiven Theorien ausgegangen wird. Dass die Lebenswelten, in denen viele unterschiedliche Handlungsmöglichkeiten existieren, schon bisher eine Berücksichtigung erfahren sollten und Unterricht nicht nur auf eine Wissensakkumulation abzielen sollte, lässt sich beispielsweise an den folgenden Zielen als Beitrag zum Bildungsbereich „Mensch und Gesellschaft“ erkennen: „Erwerb von Urteils- und Kritikfähigkeit, Entscheidungs- und Handlungskompetenz; Entwicklung von Toleranz gegenüber dem Anderen bzw. gegenüber Minderheiten; Erkennen und Bewerten von Gegebenheiten und Entwicklungen in der Arbeits- und Berufswelt; Bewertung ökonomischer Fragestellungen unter ethischen Gesichtspunkten; Einsicht in ökonomische Zusammenhänge; Aufbau eines Wertesystems zur verantwortungsbewussten Gestaltung des Lebensraums" (ebd.). Schließlich wird zumindest aus der Formulierung einiger Ziele deutlich, dass sozioökonomische Bildung pluralistisch sein soll: „Erkennen, dass in der Wirtschaft unterschiedliche Interessen aufeinander treffen und dass die Methoden des Interessenausgleichs einem Wandel unterworfen sind“ (ebd.: 78). Oder: „Erfassen subjektiver und gesamtwirtschaftlicher Probleme der Arbeitslosigkeit sowie nationaler und europäischer Lösungsansätze“ (ebd.). Damit wird deutlich, dass zwei weitere - und zusammen mit den zwei oben ausgeführten - Grundgedanken der sozioökonomischen Bildung, die hier zumindest im Ansatz gefordert werden, nämlich „Vielfalt an Handlungsoptionen" und "pluralistische Bildung" (Famulla 2019: 28f.) angesprochen werden. Somit ist nachgewiesen, dass sich alle vier ausgeführten Grundgedanken der sozioökonomischen Bildung (Famulla 2019: 25-29) bereits im GW-Lehrplan 2000 wiederfinden.

Bereits mit dem Lehrplan 1985 wurde die sozialwissenschaftliche Tradition des Unterrichtsfachs Geographie und Wirtschaftskunde begründet, und es lassen sich rund 20 Jahre später zwei Andockpunkte an die aktuelle fachdidaktische Diskussion identifizieren. Einerseits geht es um die Entfaltung und Förderung der Orientierungs-, Urteils- und Handlungsfähigkeit von jungen Menschen (Haarmann 2014: $208 \mathrm{f}$.; Kutscha 2014: 74), andererseits werden zentrale aktuelle Prinzipien der sozioökonomischen Bildung bereits im Lehrplan 2000 implizit oder explizit berücksichtigt: Subjektorientierung, Problemorientierung, Sozialwissenschaftlichkeit und Pluralität (Hedtke 2018: 95-104; ähnlich Famulla 2019: 25-29).

Bei der Gestaltung des aktuellen Lehrplans 2020 konnte erstens an die differenzierte theoretische Diskussion angeschlossen und zweitens auf empirischen Befunden aus dem Bereich der sozioökonomischen Bildung aufgebaut werden. Auf welche Art und Weise welche Ergebnisse herangezogen bzw. integriert wurden, wird in diesem Beitrag detailliert aufgearbeitet.

Es soll daher im Folgenden zwei Fragestellungen nachgegangen werden:

a) Welche Prinzipien und Gestaltungsmerkmale der sozioökonomischen Bildung wurden in den neuen GW-Lehrplan für die Sekundarstufe I integriert?

b) Welche empirischen Befunde aus der sozioökonomischen Bildung wurden auf welche Weise bei der Gestaltung des GW-Lehrplans berücksichtigt?

Für die erste Frage werden theoretische Überlegungen aus der aktuellen Diskussion in der sozioökonomischen Bildung und ihre Umsetzung im neuen GWLehrplan dargestellt. Dies betrifft die Entscheidung für eines der beiden grundlegenden Paradigmen der ökonomischen Bildung, die Kompetenzorientierung, die lebensweltlichen wirtschaftlichen Handlungsbereiche und die grundlegende Frage nach der Förderung von Mündigkeit junger Menschen (Kap. 2). Die Beantwortung der zweiten Frage beruht auf einer empirischen Studie, deren methodische Vorgangsweise (Kap. 3), einige in diesem Kontext wichtige Ergebnisse und die Berücksichtigung derer im neuen GW-Lehrplan (Kap. 4). Ein resümierender Ausblick schließt den Beitrag ab (Kap. 5).

\section{Paradigmatischer Zugang und seine Konsequenzen}

In der ökonomischen Bildung existieren im Wesentlichen zwei grundlegende, aber diametral liegende Paradigmen. Beide befinden sich am jeweiligen Endpunkt eines Kontinuums und stellen Extrempositionen dar, zwischen diesen es zahlreiche Zwischenpositionen und Mischformen gibt. Während sich das ökonomistische Paradigma monodisziplinär auf die Wirtschaftswissenschaften in monoparadigmatischer - meist neoklassischer - Ausrichtung konzentriert, bezieht sich demgegenüber die sozioökonomische Bildung inter- und transdisziplinär auf die Sozialwissenschaften und berücksichtigt dementsprechend multiparadigmatisch zahlreiche Sichtweisen, Zugänge und Modelle des Gegenstandsbereichs „Wirtschaft in der Gesellschaft“ (Hedtke 2015a: 21). Kurzgefasst: „Sozioökonomische Bildung ist die Anwendung sozialwissenschaftlicher Bildung auf den Gegenstandsbereich Wirtschaft" (Hedtke 2018: 11). Wirtschaft wird demgemäß als soziales Konstrukt und somit als gesellschaftlich eingebettet sowie gesellschaftlich konstituiert verstanden. ${ }^{3}$

\footnotetext{
Zur idealtypischen Gegenüberstellung von ökonomistischer und sozioökonomischer Bildung in Übersichten siehe zum Beispiel Hedtke 2014: 112; Hedtke 2015a: 27.
} 
Sowohl das ökonomistische als auch das sozioökonomische Paradigma ${ }^{4}$ haben sich übereinstimmend u.a. dem Ziel verschrieben, Jugendliche zu kompetenten, handlungsfähigen Menschen in ökonomisch geprägten Alltagssituationen unserer wirtschaftlich stark durchdrungenen Gesellschaft (Stichwort "Ökonomisierung“ z.B. bei Schimank \& Volkmann 2008; Engartner \& Balasundaram 2014; Hedtke 2019 und ähnlich „Intrusion" bei Bourdieu 1998) zu bilden. Doch bestehen in zentralen weiteren Merkmalen wie Bildungsgegenstand, Bildungsziel, Disziplinierung und Weltbild erhebliche Differenzen zwischen den beiden Paradigmen.

Wie die zweite, manchmal gebräuchliche Bezeichnung des ökonomistischen Paradigmas, nämlich „kategoriale ökonomische Bildung", treffend zum Ausdruck bringt, werden Inhalte deduktiv von den Bezugswissenschaften - meist überwiegend von einer am Mainstream ausgerichteten monoparadigmatischen VWL, abgeleitet und im Unterricht vereinfacht dargestellt. ${ }^{5}$ Im Kern geht es auch darum, Schülerinnen und Schüler zur Übernahme von Rollen in unserer Marktwirtschaft vorzubereiten, wobei sie allerdings die herrschende Wirtschaftsordnung unhinterfragt akzeptieren müssten. Dementsprechend müssten Menschen in diesem vorgegebenen Rahmen ihre „Spielzüge“ (Kaminski 2014: 51) machen und in „ordnungspolitischen Kategorien“, „Kreislaufzusammenhängen“ sowie in „den Kategorien der ökonomischen Verhaltenstheorie" (Kaminski 2006: 151) denken lernen, um die sogenannte „Logik des Ökonomischen" (Kaminski 2014: 50) zu verstehen. Von einem kritischen Hinterfragen des vorgefundenen Wirtschaftssystems, etwa im Hinblick auf soziale Gerechtigkeit und ökologische Nachhaltigkeit sowie von der Entwicklung von Denk- und Handlungsalternativen, ist in den Ausführungen zu ökonomistischen Ansätzen meist nur wenig zu finden. ${ }^{6}$ Zusammenfassend wird festgehalten, dass den Vorzügen der klaren Strukturierung und der guten theoretischen Durch-

\footnotetext{
Ich greife hier einzelne Gedankengänge von Fridrich 2015: $282 \mathrm{f}$. auf.

5 Vor dieser als Abbilddidaktik falsch verstandenen Wissenschaftsorientierung warnte Klafki bereits vor rund 30 Jahren in zwei Argumentationsschritten: Erstens sei die Gleichsetzung von Wissenschaftlichkeit und Einzelwissenschaft reduktionistisch. Ebenso problematisch sei zweitens die Gestaltung eines bestimmten Schulfachs aus einer bestimmten Einzelwissenschaft im Sinne eines Abbild-Verhältnisses. Vielmehr fordert er die Rückbesinnung auf eine didaktische Strukturierung eines Faches und das Heranziehen von Bezugswissenschaften zur Suche von Lösungsansätzen für Lebensprobleme (Klafki 1991: 186).

${ }^{6}$ Das trifft analog auch auf zahlreiche Wirtschaftswissenstests zu, die sowohl ein unterkomplexes Bildungsverständnis aufweisen (Uhlenwinkel 2018: 30) als auch mit neoliberalen Grundsätzen (Stieger \& Jekel 2019: 21) und neoklassischen Ansätzen (Rumpold \& Fuhrmann 2016: 125) in Verbindung stehen.
}

dringung des ökonomistischen Paradigmas durch die Bezugswissenschaft VWL erhebliche Nachteile für den Unterricht entgegenstehen: hohes Abstraktionsniveau, geringe Einbindungsmöglichkeit von Vorerfahrungen der Lernenden, kaum Berücksichtigung von Interessen, sowie oft geringer Lebenswelt- und Alltagsbezug aufgrund der deduktiven Ableitung bereits prädeterminierter, hochgradig strukturierter Themen. Das hier zum Ausdruck kommende Menschenbild ist einerseits der Homo oeconomicus als Nutzenmaximierer, der zu uneingeschränktem rationalen Verhalten fähig ist und über vollständige Information über alle Märkte verfügt sowie andererseits das „unternehmerische Selbst", das optimale Kosten-Nutzen-Kalküle bei knappen Mitteln nicht nur auf ökonomische Situationen, sondern flexibel und unternehmerisch auf die eigene Person anwendet (Famulla 2019: 20-25).

Die skizzierten Aspekte der ökonomistischen Bildung werden nun ausgewählten Prinzipien der sozioökonomischen Bildung in Österreich gegenübergestellt, die im „Positionspapier Sozioökonomische Bildung" zusammengefasst wurden (Fridrich \& Hofmann-Schneller 2017: 56). Die Schüler/innen- und Lebensweltorientierung ist im ökonomistischen Paradigma nicht durchgehend gewährleistet, wenn etwa von Modellen ausgegangen wird, die nachvollzogen werden sollen. „Kritische Zugänge durch Reflexion des praktizierten Wirtschaftens auf Haushalts-, Unternehmens- und Gesellschaftsebene einschließlich der Interessen- und Machtkonstellationen im Sinne eines Zugangs zur politischen Bildung" (ebd.) werden im ökonomistischen Paradigma kaum gefördert, sondern vielmehr der Nachvollzug der vorgefundenen Wirtschaftsordnung. Wenn Unterricht nur auf neoliberalen Zugängen und neoklassischen Grundsätzen aufbaut, bleiben inhaltliche Mehrperspektivität und Pluralismus auf der Strecke. Was für die sozioökonomische Bildung im Speziellen gilt, wurde von der GW-Fachdidaktik im Speziellen im fachdidaktischen Grundkonsens festgehalten. Dort heißt es beispielsweise im Rahmen der didaktischen und methodischen Prinzipien: „Orientierung an Qualifikationsansprüchen, die zur Lösung komplexer Problemstellungen befähigen (Kompetenzorientierung); Orientierung am Prinzip der inhaltlichen Mehrperspektivität und der Kontingenz; Politische Bildung auf Basis gesellschaftskritischer Reflexionen" (zusammenfassend Pichler et al. 2017: 62).

Fazit 1: Der Vergleich des ökonomistischen Paradigmas mit dem sozioökonomischen Paradigma macht deutlich, dass das erstgenannte weder zu den Intentionen der durch die GW-Lehrpläne 1985 und 2000 grundgelegte sozialwissenschaftliche Tradition des Faches Geographie und Wirtschaftskunde passt, noch zur vertretenen sozioökonomischen Bildung im Spe- 
ziellen, noch zur fachdidaktischen Ausrichtung des Faches im Allgemeinen. Dieses ökonomistische Paradigma wurde daher für den neuen GW-Lehrplan 2020 nicht berücksichtigt.

Eine hervorragende Anschlussfähigkeit findet hingegen die lebenswelt- und subjektorientierte sozioökonomische Bildung, in der problemorientierte Realsituationen aus den Lebenswelten von Kindern und Jugendlichen zum Ausgangspunkt von Unterricht genommen werden. Somit erfolgt die Auswahl und Schwerpunktsetzung von Themen induktiv aus dem gesellschaftlichen Handlungskontext (Fischer \& Zurstrassen 2014: 10) und deren Bearbeitung unter Einbindung fundierter Erkenntnisse aus Human- und Physiogeographie, Kartographie und Medienbildung, ökonomischer und politischer Bildung, Bildung für nachhaltige Entwicklung, Verbraucher/innenbildung und -forschung, Bildungs- und Berufsorientierung, Ökologie sowie ggf. aus weiteren Sozial- und Wirtschaftswissenschaften. Zudem sollen Themen- und Problemstellungen im Wirkungsgefüge Gesellschaft - Wirtschaft - Politik - Umwelt in räumlichen und zeitlichen Kontexten bearbeitet werden, was zu Beginn der Bildungs- und Lehraufgabe sowie mehrmals in den Kompetenzformulierungen expressis verbis so ausgedrückt wird. Der prinzipielle Zugang ist somit multidisziplinär und inhaltlich mehrperspektivisch.

Die geförderten Kompetenzen in den Sphären Privathaushalt, Konsum, Arbeitswelt und Gesellschaft dienen nicht nur zur viel zitierten Bewältigung von ökonomisch geprägten Alltagssituationen, sondern beinhalten selbstverständlich auch die Aufforderung zum kritischen Nachdenken sowie selbstbewussten, verantwortungsvollen und solidarischen Mitgestalten von Wirtschaft und Gesellschaft. Es darf daher nicht nur innerhalb des vorgefundenen Wirtschaftssystems gedacht und gehandelt werden, sondern auch über dieses reflektiert sowie Denk und Handlungsalternativen entworfen werden.

Fazit 2: Die in den Vorgängerlehrplänen 1985 und 2000 implizit zum Ausdruck kommende Tradition der sozioökonomischen Bildung wurde im neuen GW-Lehrplan 2020 als weiterhin anschlussfähig erkannt und im Sinne der Lebenswelt-, Subjekt- und Problemorientierung sowie in Bezug auf Pluralität, Multidisziplinarität sowie inhaltliche Mehrperspektivität fortgeführt und vertieft.

Hierbei kommt der Förderung von wirtschaftsbezogenen Kompetenzen in allen kognitiven Kompetenzstufen (Anderson \& Krathwohl 2001: $61 \mathrm{f}$.) bzw. gleichbedeutend - in den drei Anforderungsbereichen Reproduktion (Wiedergeben, Verstehen), Reorganisation (Anwenden, Analysieren) und Reflexion (Bewerten, Gestalten) eine besondere Bedeutung zu (Bun- desministerium für Bildung und Frauen 2012: 13f.) - und dies bezogen auf das Wirkungsgefüge „Gesellschaft - Politik - Wirtschaft - Umwelt“. Diese kognitiven Fähigkeiten und Fertigkeiten lassen sich auf jeder Stufe in vier Dimensionen aufgliedern (Anderson \& Krathwohl 2001: 46; Fridrich 2019: 78): Faktenwissen umfasst beispielsweise Terminologie, Details, Sachverhalte etc., wobei Wissen im Alltagssprachgebrauch oft synonym mit Faktenwissen gleichgesetzt wird. Im Konzeptwissen werden inhaltliche Prinzipien, Modelle, Theorien etc. subsummiert, was mit der Einführung der Basiskonzepte in den GW-Lehrplan der AHS-Oberstufe implementiert wurde (Hinsch et al. 2014: 51) und mit der Entwicklung von sogenannten „zentralen fachlichen Konzepten“" nun auch für die Sekundarstufe I umgesetzt wurde (siehe unten). Prozesswissen bedeutet den Einsatz fachspezifischer Fertigkeiten, Techniken, Methoden etc., die einen Ausgangspunkt für den Einsatz ökonomisch orientierter, methodischer Kompetenzen bilden. Unter metakognitivem Wissen wird Nachdenken über Lernstrategien, Reflexion eigener Lernprozesse etc. verstanden, wobei diesem im deutschen Sprachraum generell zu wenig Beachtung geschenkt wird.

Fazit 3: Neben den expliziten Hinweisen auf die erforderliche Kompetenzorientierung in der Bildungsund Lehraufgabe des Lehrplans wurde bei der Kompetenzformulierung von der 5. bis zur 8. Schulstufe auf Formulierungen auf allen drei Anforderungsbereichen und in allen vier Wissensdimensionen bezüglich des Wirkungsgefüges „Gesellschaft - PolitikWirtschaft - Umwelt" geachtet.

Im Hinblick auf das oben angeführte Konzeptwissen wurde vom Bildungsministerium der Auftrag an jede ministerielle Arbeitsgruppe gegeben, sogenannte „zentrale fachliche Konzepte“ zu identifizieren und zu erläutern. Für Geographie und Wirtschaftskunde war diese Entwicklung ein vergleichsweise leichtes Unterfangen, weil bereits auf zwei vorliegende Arbeiten zurückgegriffen werden konnte. Einerseits lagen bereits die mit dem GW-Lehrplan der AHS-Oberstufe formulierten „Basiskonzepte“ vor (Hinsch et al. 2014: 52-55), andererseits wurde vor einiger Zeit ein konkreter Diskussionsvorschlag für Basiskonzepte für GW in der Sekundarstufe I entwickelt (Fridrich 2016). Unter Verwendung beider Quellen, weiterer Publikationen und Einbeziehung fachdidaktischer Überlegungen wurden folgende fünf fachliche Konzepte entwickelt und erläutert: Gemeinsamkeiten und Unterschiede, Vernetzung und Veränderung, Interessen und Macht, Werte und Identitäten, Maßstabsebenen und Raum. Diese zentralen fachlichen Konzepte für die Sekundarstufe I sind in ihrer Anzahl auf wesentliche Aspekte fokussiert, in ihrer Begrifflichkeit verständlich, weisen 
ein einheitliches, an die Altersstufe angepasstes Abstraktionsniveau auf und beziehen sich gleichermaßen auf geographische, wirtschaftliche, ökologische und politische Bildung, was aus der Formulierung dieser Konzepte im Lehrplanentwurf vom 15.11.2019 deutlich wird. Beispielsweise soll im Folgenden das zentrale fachliche Konzept „Vernetzung und Veränderung“" zitiert werden, um zu zeigen, dass die eben ausgeführten Kriterien umgesetzt wurden: „Menschen leben und wirtschaften nicht isoliert, sondern vernetzt. Daher haben menschliche Handlungen an einem Ort oft Auswirkungen auf andere, manchmal weit entfernte Orte und Menschen. Vernetzung und Veränderung sind somit verbunden - in den Bereichen Gesellschaft, Wirtschaft, aber auch in Mensch-Umwelt Beziehungen. Beispielsweise werden zahlreiche wirtschaftliche Zusammenhänge durch Vernetzung erklärbar, etwa Ströme von Gütern, Dienstleistungen, Informationen und Geld. Dies geschieht sowohl in Marktbeziehungen (z. B. Arbeitsmarkt und Gütermärkte) als auch durch marktferne wirtschaftliche Aktivitäten (z.B. unbezahlte Pflege und Erziehung im Haushalt)“.

Fazit 4: Die fünf zentralen fachlichen Konzepte von $G W$ stellen im Sinne von fundamentalen Ideen bzw. "Big Concepts" die Prinzipien des Faches in der Sekundarstufe I dar, beziehen sich stets auf das Wirkungsgefüge "Gesellschaft - Wirtschaft - Politik - Umwelt", sind quantitativ überschaubar und qualitativ durchschaubar.
Wenn Emanzipation (Selbstbestimmung) und Partizipation (Mitbestimmung) als Leitbilder für den Unterricht und als wesentliche Anliegen von Schule verstanden werden, durch die junge Menschen gesellschaftliche Mündigkeit durch das Erschließen von wirtschaftlichen und politischen Zusammenhängen erlangen, sind Orientierungs-, Urteils- und Handlungskompetenz unverzichtbare übergeordnete Kompetenzen (Haarmann 2014: 208 f.; Kutscha 2014: 74; ähnlich Hedtke 2018: 33 als „sozioökonomische Leitkompetenzen“). Dieses elaborierte Konzept übergeordneter Kompetenzen stammt aus der sozioökonomischen Bildung und wurde für einen GW-Unterricht in adaptierter und weiterentwickelter Form unter Einbeziehung fachdidaktischer Überlegungen fruchtbar gemacht, in dem verschiedenste Aspekte des Wirkungsgefüges „Gesellschaft - Wirtschaft - Politik - Umwelt" auf unterschiedlichen Maßstabsebenen bearbeitet werden. Dieses Kompetenzmodell ist in Übersicht 1 visualisiert.

Fazit 5: Das Kompetenzmodell des Unterrichtsgegenstandes Geographie und Wirtschaftliche Bildung folgt im GW-Lehrplan 2020, unter Einbeziehung fachdidaktisch und fachwissenschaftlich begründeter und fundierter Methoden, Inhalte sowie Konzepte, den sozioökonomischen Leitkompetenzen: Orientierungs-, Urteils- und Handlungskompetenz.

Diese Orientierungs-, Urteils- und Handlungskompetenzen sollen aus der Perspektive der sozioökono-

\section{Gesellschaft}

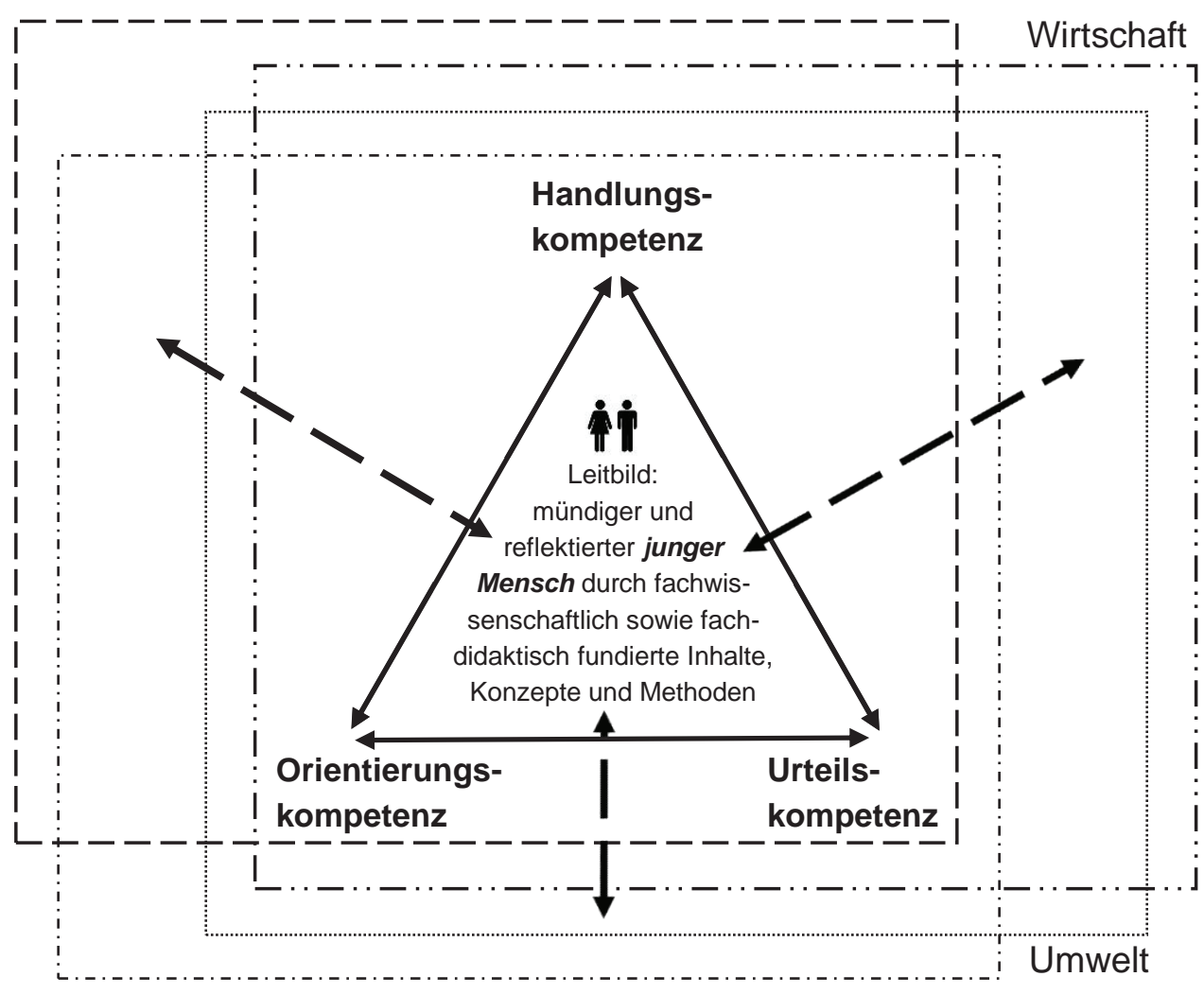

Politik
Übersicht 1: Gemäß dem neuen GW-Lehrplan visualisiertes Kompetenzmodell (Quelle: eigene Darstellung C. Fridrich) 
mischen Bildung in den folgenden drei überlappenden Wirtschaftsbereichen des Alltags entwickelt und gefördert werden, wobei Geld und Finanzen eine Querschnittsmaterie für alle drei Bereiche darstellt: 1. Privathaushalt und Konsum, 2. Arbeitswelt, 3. Gesellschaft (Sitte 2001: 546-51; Tenfelde \& Schlömer 2012: 440 f.; Fridrich 2019: 79 f.).

Privathaushalte stellen die "Schaltstellen“ der Gesellschaft und die „Basiseinheiten der Wirtschaft“ dar (Thiele-Wittig 2003), in denen Güter und Dienstleistungen sowohl produziert als auch konsumiert werden, Geld eingenommen, ausgegeben und angelegt wird sowie Verträge unterschiedlicher Art abgeschlossen werden. Durch eine zunehmende Komplexität des wirtschaftsgeprägten Alltags etwa bei Altersvorsorge, Gütesiegeln, Nachhaltigkeit, Versicherungen etc. (Engartner \& Krisanthan 2014b: 168 f.), durch steigende Erfordernisse der Eigenproduktion und bei Konsumprozessen, besonders im Dienstleistungssektor (zum Präsumieren bei partizipativen Internetplattformen, Bankgeschäften, Reiseplanungen etc. siehe z. B. den Sammelband von Hellmann und Blättel-Mink 2010), und durch erforderliche Abstimmungsprozessen bei der Bedürfnisbefriedigung der Haushaltsmitglieder durch materielle und immaterielle Versorgung (Piorkowsky 2012: 437 f.) ist eine fundierte Bearbeitung des eng verflochtenen Handlungsbereiches Privathaushalt und Konsum im Unterricht unabdingbar.

Die Arbeitswelt ist für Schülerinnen und Schüler in mehrfacher Weise ein bedeutendes Handlungsfeld, das bereits ab der Primarstufe durch Realbegegnungen und grundlegende Erkenntnisse erschlossen, in der Sekundarstufe I mit Bildungs- und Berufsorientierung sowie relevanten Themen aus der Arbeitswelt fortgesetzt und schließlich in der Sekundarstufe II durch vertiefte Analyse und Bewertung von komplexen Themen- und Fragestellungen vertieft werden soll (Fridrich 2019: 79). Im Konkreten geht es darum, Betriebe und Unternehmen in altersadäquater Weise als wesentliche Orte wirtschaftlicher Leistungserstellung zu verstehen. Die Zugänge sollen im Sinne der Mehrperspektivität die Sichtweisen und Standpunkte von Arbeitnehmerinnen und Arbeitnehmern einerseits sowie Arbeitgeberinnen und Arbeitgebern andererseits umfassen und arbeitssituationsbezogene und gesellschaftspolitische Grundwerte vermitteln (Engartner \& Krisanthan 2014b: 168). Individuelle Sinnstiftung und gesellschaftliche Integration durch Arbeit, Kreativität, Eigeninitiative und Mitgestaltungsoptionen werden nicht nur theoretische Aspekte bleiben, sondern als Anknüpfungspunkte für unterrichtliche Bearbeitungen dienen.

Als Zivilbürgerin und Zivilbürger sind wirtschaftende Menschen Teil der Gesellschaft. Neben dem sicherlich bedeutenden materiellen Beitrag für das
Gemeinwesen (z. B. Staat) durch die Bezahlung von Beiträgen und Steuern und das Beziehen von Leistungen durch das Individuum (Engartner \& Krisanthan 2014b: 169), sollte die Erkenntnis gewonnen werden, dass Wirtschaft nicht naturgesetzlich funktioniert, sondern in die Gesellschaft eingebettet ist und durch gesellschaftliche Konstitutionsleistungen produziert und reproduziert wird (Fridrich 2019: 79 f.). Neben der Bezugnahme auf Aufgaben und Zusammenwirken von Individuen, Unternehmen, des Staates und anderen Wirtschaftsteilnehmer/innen geht es z.B. im 3. Kompetenzbereich des Lehrplanentwurfs vom 15.11.2019 um ausgewählte wirtschaftliche, soziale und ökologische Kenngrößen, um wirtschaftliche Entwicklungen sowie um deren Auswirkungen. Je nach Machtausstattung von Personen und Gruppen kann Wirtschaft und Gesellschaft mehr oder weniger intensiv mitgestaltet werden. Auch zivilgesellschaftliches ökonomisches Handeln findet in räumlichen und historischen Kontexten statt und steht in enger Beziehung zu gesellschaftlichen Normen und Werten, aber auch zu psychologischen Aspekten (Engartner 2019a: 21).

In allen drei eben ausgeführten und einander überlappenden Handlungsdimensionen von Wirtschaft in Lebensweltkontexten sind Selbstbestimmung, Mitbestimmung und Solidarität gefragt, was die sozioökonomischen Leitkompetenzen Orientierungs-, Urteils- und Handlungskompetenz erfordert. Diese zu entwickeln und zu fördern ist eine anspruchsvolle Aufgabe und angemessener als einen Menschen als homo oeconomicus zu trainieren: „Der homo oeconomicus, der alles Tun und Trachten - von der Aufnahme des Studiums über die Berufswahl bis hin zur Familiengründung - unter den ökonomischen Vorbehalt des ,Sich-Rechnen-Müssens's stellt, darf nicht als Leitbild in Bildungsprozessen fungieren, kann allenfalls als Abbild individuelles Verhalten modellieren. Andernfalls droht die Semifiktion des homo oeconomicus eines Tages eine Reifikation zu erfahren, sprich: aus der Modellwelt herauszutreten und in der realen Welt Fuß zu fassen, sodass die an Eigennutz und Zweckrationalität orientierte Individualität die von Gemeinsinn und Kollektivität geprägte Sozialität endgültig usurpiert“ (Engartner 2019b: 96).

In Übersicht 2 wird zusammenfassend dargestellt, in welche Kompetenzen die oben ausgeführten lebensweltlichen Wirtschaftsbereiche in den einzelnen Schulstufen integriert wurden. Kompetenzen der Querschnittsmaterie Geld wurden je nach Schwerpunktsetzung jeweils einem der Bereiche zugeordnet. Prinzipiell wurden die Kompetenzen nach aufsteigender Komplexität von der 5. bis zur 8. Schulstufe formuliert. Die Nummern geben die jeweiligen durchnummerierten Kompetenzen auf der entsprechenden 
Schulstufe im Lehrplanentwurf vom 15.11.2019 an. Es erfolgte zudem eine überwiegende Zuordnung zu einem der drei Bereiche, außer wenn in der Formulierung der jeweiligen Kompetenz eindeutige Überlappungen festgestellt wurden und somit für zwei Wirtschaftsbereiche in Frage kam.

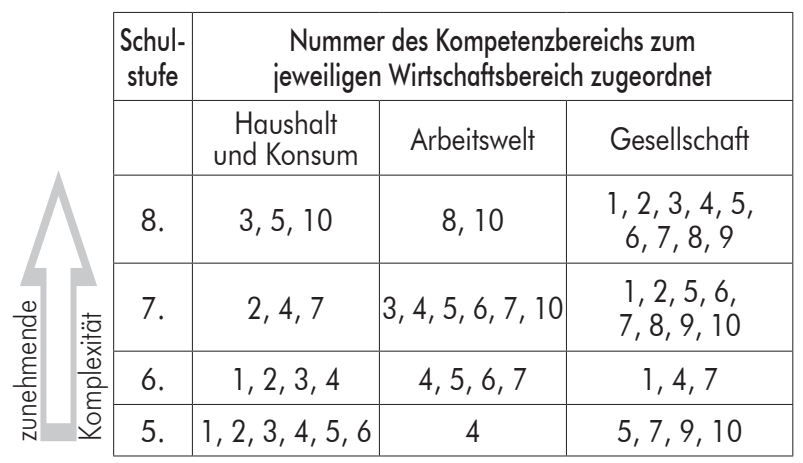

Übersicht 2: Zuordnung der Kompetenzen (Nummer) zu den lebensweltbezogenen Wirtschaftsbereichen, getrennt nach Schulstufe im neuen GW-Lehrplan (Quelle: eigene Darstellung C. Fridrich)

Am Beispiel der altersadäquaten Zielstellungen für den Themenbereich Geld soll nun das Prinzip der zunehmenden Komplexität der Kompetenzen demonstriert werden. In der 5. Schulstufe kommt Geld in einfachen Haushaltskontexten vor. Dort heißt es im Kompetenzbereich „Leben und Wirtschaften im eigenen Haushalt“ in Kompetenz 2: „Das Produzieren und Konsumieren sowie den Umgang mit Geld im Haushalt analysieren". Aus ökonomistischer Sichtweise würde dies bedeuten, Geld in seinen Funktionen als Zahlungsmittel, Wertmesser und Wertaufbewahrungsmittel durchzunehmen. Die sozioökonomische Bildung geht weiter ${ }^{7}$. Geld wird hier nicht nur zusätzlich als Statussymbol betrachtet, mit dem Einfluss und Ansehen verstärkt werden; es wird auch der persönliche Umgang mit Geld diskutiert und reflektiert. Dabei geht es um die subjektive Bedeutung von Geld ebenso wie um Einnahmen und Ausgaben sowie deren Relation zueinander in lebensweltlichen Kontexten. Charakteristische Fragen dazu wären etwa: Was geschieht, wenn du auf Dauer mehr ausgibst als einnimmst? Welche Möglichkeiten hast du, wenn du immer wieder mehr Geld einnimmst als ausgibst?

Im Zusammenhang mit Angebot, Nachfrage und Preis nimmt Geld in der 6. Schulstufe im Kompetenzbereich „Vernetztes Wirtschaften - Produzieren und Konsumieren von Gütern und Dienstleistun-

\footnotetext{
Hier werden bewusst kontrastierend ökonomistische und sozioökonomische Zugänge und Umsetzungen dichotomisierend gegenübergestellt, um die jeweiligen Schwerpunkte und grundlegenden Intentionen herauszuarbeiten. In der Praxis existieren unzählige Mischformen zwischen den beiden idealtypisch verorteten Polen eines Kontinuums.
}

gen" in Kompetenz 7 eine prominente Stellung ein. Die Formulierung lautet: „Ideen zur Erzeugung von Gütern und Dienstleistungen unter Analyse von Angebot, Nachfrage und Preis entwickeln, umsetzen und die Auswirkungen reflektieren“. Eine Vorgangsweise aus ökonomistischer Perspektive wäre hier ein einfaches Beispiel mit Angebot- und Nachfragekurve zu erstellen, was nicht die Intention dieser Kompetenz des Lehrplans ist. Gemeint ist an dieser Lehrplanstelle vielmehr ein sozioökonomischer Zugang, mit dem Lehrende gemeinsam mit ihren Lernenden anhand eines einfachen, selbst gewählten Beispiels das Zusammenwirken von Angebot, Nachfrage und Preis bei der Erzeugung von einfachen Gütern und Dienstleistungen aus ihrem Alltag überlegen und schließlich umsetzen. Ein Beispiel dazu wäre etwa, Aufstrichbrote für ein Schulfest oder für einen Elternsprechtag herzustellen und dann zu verkaufen. Folgende Fragen könnten dabei aufgeworfen werden: Wie viel kostet das Material insgesamt und welche Gesamtsumme ist daraus erzielbar? Ergibt sich ein Gewinn oder Verlust? Welcher Unterschied besteht zwischen Einnahmen und Gewinn? Wie ändert sich die Berechnung, wenn die Arbeitszeit für Herstellung und Verkauf integriert werden würde? Wie kann man günstiger produzieren? Welcher Verkaufspreis pro Brot ist erzielbar? Was kann mit dem erzielten Geld unternommen werden?

In der 7. Schulstufe ist Geld in zwei Kompetenzen integriert, nämlich einmal im Zusammenhang mit der Arbeitswelt und ein anderes Mal im Kontext volkswirtschaftlicher Aspekte, wodurch eine Zunahme der Komplexität ab der 5. Schulstufe deutlich wird. Im Kompetenzbereich „Bildungswege und Arbeitswelten" wird in Kompetenz 4 auf Geld in Form von Einkommen fokussiert: „Unbezahlte und bezahlte Arbeit, Arbeitslosigkeit, Höhe und Verwendung des Einkommens [...]“. Im Kompetenzbereich „Akteure und Entwicklungen am Wirtschaftsstandort Österreich" wird auf volkswirtschaftliche Aspekte Bezug genommen: „Ausgewählte wirtschaftliche Entwicklungen Österreichs wie Wirtschaftswachstum, Inflation und Arbeitslosigkeit beschreiben und deren persönliche sowie gesellschaftliche Auswirkungen analysieren“. Für beide Kompetenzen würden sich Differenzen bei einer ökonomistischen im Vergleich zu einer sozioökonomischen Vorgangsweise erkennen lassen. Zunächst zum Bereich der Höhe und der Verwendung des Einkommens: Während in ökonomistischen Zugängen eher auf einfache und lineare Wenndann- oder Je-desto-Beziehungen abgezielt werden würde, wie etwa "Je höher das Bildungsniveau, desto besser der Beruf, desto höher das Einkommen " und fehlendes Geld mit Faulheit assoziiert werden würde, sind die Fokussierungen im sozioökonomischen Paradigma wesentlich breiter. Hier können in einer mehr- 
perspektivischen Sichtweise Gründe von Armut und Reichtum erschlossen werden sowie die Erkenntnis gewonnen werden, dass auch viel und schwer arbeitende Menschen nicht automatisch reich sein müssen. Es wird zudem die Bedeutung der Ausbildung und eines ,geeigneten “ Berufs für Lebenszufriedenheit und Einkommen anhand von Ideen, Erfahrungen und Alltagsvorstellungen der Lernenden und zwar für ihr eigenes Leben thematisiert. Zudem kann diskutiert werden, welche Rolle Geld im Leben der Jugendlichen spielt. Geht es darum, möglichst viel Geld zu verdienen und auszugeben oder um Vorstellungen und Umsetzungsversuche von einem guten Leben? Im anderen Kompetenzbereich der 7. Schulstufe geht es um Inflation und Geldwert. Aus ökonomistischer Sicht wird dieses Thema durch Erläuterungen von Bedeutung, Messung, Arten und Auswirkungen der Inflation, einem möglichen Datenvergleich zwischen Staaten sowie einer abschließenden Definition durchgenommen. Gemäß dem Paradigma der sozioökonomischen Bildung eröffnen sich zusätzliche Optionen. Zunächst wird von lebensweltlichen Erfahrungen der Lernenden ausgegangen und beispielsweise die jährliche Verteuerung der Eiskugeln analysiert. Folgende Fragen werden dabei aufgeworfen: Warum gibt es diese Verteuerungen überhaupt? Wem nützen sie? Wer hat Nachteile davon? Welche anderen Fallbeispiele lassen sich für den ständigen Anstieg des Preisniveaus finden? Werden einzelne Güter oder Dienstleistungen auch billiger, und wenn ja, warum? Was bedeutet Inflation für Menschen mit einem Sparguthaben bzw. mit einem Kredit? Im Zuge der Erörterungen ist es selbstverständlich erforderlich, auf wissenschaftliche Ergebnisse und Modelle in alters- und schüler/innengemäßer Form zurückzugreifen und diese in den Unterricht einzubinden. Doch muss die Bedeutung der Inflation für das eigene Leben im Vordergrund stehen.

Schließlich werden der Euro als gemeinsame Währung und der freie Kapitalverkehr als wichtige Elemente der Europäischen Union im Kompetenzbereich „Europa und Europäische Integration“ der 8. Schulstufe in der Kompetenz 5 angeführt: „Die Notwendigkeit gemeinsamer Werte wie Friedenserhaltung, Demokratie oder Freiheit begründen und EU-Projekte wie die vier Freiheiten, den Euro, Qualitätsstandards, Verbraucherschutz und Regionalentwicklung analysieren sowie deren Bedeutung für das eigene Leben reflektieren". Euro und freier Kapitalverkehr treten hier einerseits in komplexeren volkswirtschaftlichen und supranationalen Zusammenhängen auf, andererseits im Haushaltskontext, wenn es um die Bedeutung für das eigene Leben geht. Auch hier existieren zwei grundlegend verschiedene Zugangsweisen. In ökonomistischen Annäherungen wird besondere Betonung auf die historische Entwicklung der Eurozone, auf
Vor- und Nachteile der gemeinsamen Währung auf einer allgemeinen Ebene sowie auf EU-Institutionen im Zusammenhang mit dem Euro gelegt. Im Zuge der sozioökonomischen Bildung wird von lebensweltlichen Erfahrungen wie etwa eigenem Reisen oder Einkaufen der Eltern im Internet ausgegangen. Wo lebensweltliche Zugänge nicht direkt anschließen können, wie etwa der Bedeutung des Euros für den Handel innerhalb der Eurozone, werden anschauliche Fallbeispiele herangezogen werden müssen. Doch auch hierbei ist wiederum auf inhaltliche Mehrperspektivität zu achten.

Fazit 6: Die im neuen GW-Lehrplan formulierten Kompetenzen lassen sich nach dem Prinzip der zunehmenden Komplexität den einzelnen lebensweltorientierten Wirtschaftsbereichen Privathaushalten und Konsum, Arbeitswelt, Gesellschaft zuordnen und decken damit auf der Inhaltsebene altersadäquat zentrale Bereiche der sozioökonomischen Bildung ab.

Für zwei Aspekte der Gestaltung des GW-Lehrplans 2020 - und zur Beantwortung der zu Beginn formulierten zweiten Fragestellung - wurde auf Ergebnisse aus einer empirischen Studie über den Lehrplan 2000 zurückgegriffen. Es sind dies erstens der relative Anteil von geographischen und wirtschaftskundlichen Inhalten sowie deren Vernetzung an der reinen GWUnterrichtszeit und zweitens die Reihung von GWThemenkreisen nach ihrer eingeschätzten Wichtigkeit durch GW-Lehrpersonen.

\section{Relevanz und Bedeutung wirtschafts- kundlicher Themenfelder aus der Perspektive von Lehrkräften}

\subsection{Methodische Vorgehensweise der Studie}

Für diesen relevanten Teil der empirischen Studie wurden GW-Lehrpersonen in der AHS-Unterstufe und an der damaligen Hauptschule, die nun der Neuen Mittelschule entspricht, befragt. Im zweiten angeführten Schultyp unterrichten neben Lehrpersonen mit einem Studienabschluss in Geographie und Wirtschaftskunde auch Lehrerinnen und Lehrer ohne entsprechende Lehramtsprüfung, also „fachfremd“ bzw. „ungeprüft". Dies betrifft nicht nur das Fach GW. Es wurde in dieser Studie auch diese Personengruppe der ungeprüft GW Unterrichtenden befragt, weil diese einen bedeutenden Prozentanteil der gesamten Lehrpersonen ausmachen und damit zur Schulrealität beitragen. Die Studie wurde zunächst auf die Sekundarstufe I in Wien beschränkt, hier jedoch eine Totalerhebung vorgenommen. 
Nach der Erstellung des Fragbogens auf Basis theoretischer Überlegungen, fanden Prätests, Auswertungen und Modifizierungen statt (siehe dazu und zum Folgenden im Detail Fridrich 2015: 284-286). Die Datenerhebung dieser bislang nur in Auszügen publizierten Studie fand von Februar bis April 2011 statt. Aufgrund der gewählten Vorgangsweise war die Rücklaufquote der verwertbaren Fragebögen mit 65,7\% sehr hoch: Die Fragebogen wurden zu einem vereinbarten Zeitpunkt in die Schule gebracht, Interessierten persönlich überreicht und meist am gleichen Tag bzw. am Folgetag wieder persönlich abgeholt. Anschließend wurden sowohl die Daten aus den offenen als auch aus den geschlossenen Fragestellungen aller verwertbaren Fragebogen ( $\mathrm{n}=527)$ in eine Exceltabelle übertragen sowie nach einer Datenkontrolle und Datenbereinigung in ein Gesamt-File zur weiteren Auswertung übergeführt.

\subsection{Relativer Anteil von geographischen und wirtschaftskundlichen Inhalten sowie deren Vernetzung an der reinen GW-Unterrichtszeit}

Für dieses Thema wurde gemäß den forschungsleitenden Fragestellungen in Bezug auf den Lehrplan 2000 folgende Hypothese formuliert: Der Anteil wirtschaftskundlich dominierter Themenbereiche ist gemäß GW-Lehrplan in der 3. Klasse deutlich höher als in der 1. und 2. Klasse - dies hat jedoch keinen Zusammenhang mit einem größeren Anteil von wirtschaftskundlichen Inhalten an der GW-Unterrichtszeit.

Die zugeordnete Fragebogenfrage lautete: „Wie viel Prozent Ihrer reinen GW-Unterrichtszeit verwenden Sie jeweils im Schnitt für geographische Inhalte, für wirtschaftskundliche Inhalte und für deren bewusste Vernetzung?" Für jede Klasse war der selbst einzuschätzende Prozentanteil anzugeben.

Eine Analyse des in Übersicht 3 eingetragenen arithmetischen Mittels zeigt, dass von der 1. bis zur 4 . Klasse sowohl die Anteile der wirtschaftskundlichen Inhalte als auch die bewusste Vernetzung von geographischen und wirtschaftskundlichen Inhalten gemäß den Aussagen der befragten Lehrpersonen kontinuierlich steigen. Der mit höherer Schulstufe zunehmende Anteil wirtschaftskundlicher Inhalte im Lehrplan wirkt sich nach Angaben der befragten Lehrpersonen mit einer höheren Unterrichtszeit für wirtschaftskundliche Themen in ihrem Unterricht aus.

Mit einer Varianzanalyse zwischen den Anteilen der Unterrichtszeit für die drei Bereiche geographische Inhalte, wirtschaftskundliche Inhalte und bewusste Vernet$z u n g$ - und dies für die Klassen 1 bis 4 - wurde festgestellt, dass die Unterschiede im Anteil geographischer und im Anteil wirtschaftskundlicher Inhalte sowie in der bewussten Vernetzung zwischen den Schulstufen signifikant sind. Für geographische Inhalte: $\mathrm{F}(2,55$; 1004,87) = 316,16; $\mathrm{p}<0,01$; für wirtschaftskundliche Inhalte: $\mathrm{F}(2,77 ; 1090,23)=180,51 ; \mathrm{p}<0,01$; für bewusste Vernetzung: $F(2,32 ; 914,90)=91,31 ; \mathrm{p}<0,01$.

\section{Prozentanteile von Inhalten}

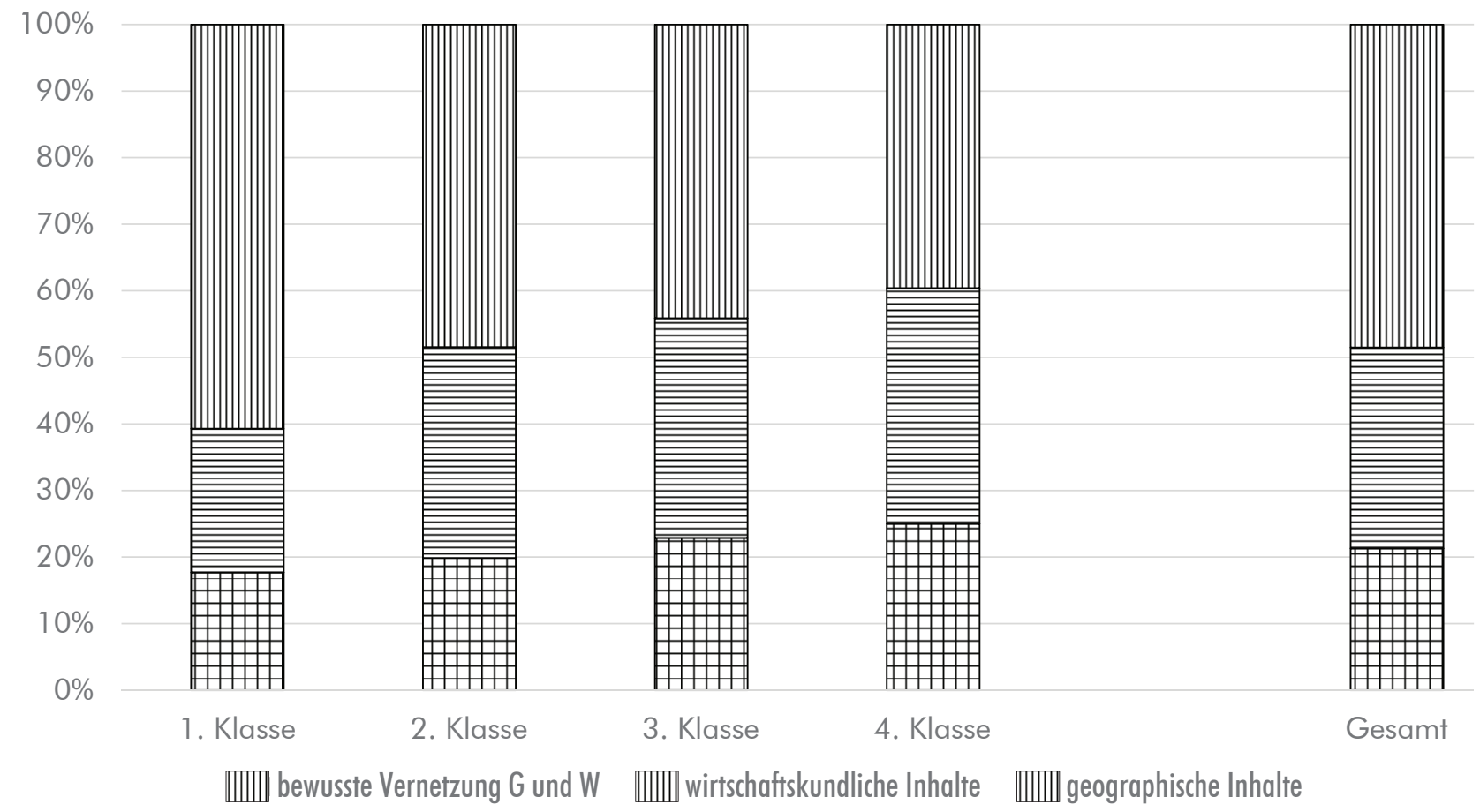

Übersicht 3: Relativer Anteil von geographischen Inhalten, wirtschaftskundlichen Inhalten und deren Vernetzung an der reinen GW-Unterrichtszeit bezogen auf den „alten“ GW-Lehrplan 2000 (Quelle: eigene Darstellung C. Fridrich) 
Das bedeutet, dass von der 5. bis zur 8. Schulstufe sowohl die Zeit für die Bearbeitung wirtschaftskundlicher Inhalte als auch die Zeit für die bewusste Vernetzung von geographischen und wirtschaftskundlichen Inhalten signifikant über die Schulstufen hinweg ansteigt, während die Zeit für die Erarbeitung geographischer Inhalte im gleichen Zeitraum signifikant abnimmt. Deswegen wird die Nullhypothese verworfen.

Doch es bestand Verbesserungsbedarf für den neuen Lehrplan, der aus diesen empirischen Daten ableitbar war. Diese Verbesserung wurde in folgenden beiden Bereichen erzielt: Erstens wurden nun die Kompetenzen auf das Wirkungsgefüge Gesellschaft Wirtschaft - Politik - Umwelt bezogen vernetzt formuliert. Zweitens existieren mit dem GW-Lehrplan 2020 keine Kompetenzen und Kompetenzbereiche mehr, die ausschließlich auf geographische Zielstellungen ausgerichtet sind.

Fazit 7: Eine noch bessere Vernetzung von geographischen und wirtschaftskundlichen Kompetenzen im neuen Lehrplan im Vergleich zum Lehrplan 2000 sowie ein in allen Schulstufen durchgehend hoher Anteil von wirtschaftskundlich orientierten Kompetenzen wurde umgesetzt, weil dies bei GWLehrpersonen einen direkten Einfluss auf die von ihnen eingeschätzte Gestaltung des Unterrichts zeigte. Über den gesamten GW-Lehrplan 2020 betrachtet, beziehen sich die Kompetenzen durchwegs vernetzt auf das Wirkungsgefüge Gesellschaft - Wirtschaft Politik-Umwelt.

\subsection{Reihung von GW-Themenkreisen nach ihrer eingeschätzten Wichtigkeit}

Aufgrund von Vermutungen und theoretischen Überlegungen wurde in Bezug auf den Lehrplan 2000 folgende Hypothese aufgestellt: Wirtschaftskundlich dominierte Themenkreise des GW-Lehrplans werden von den Lehrpersonen als weniger wichtig eingeschätzt als andere Themenkreise des Lehrplans.

Zugeordnete Fragebogenfrage: „Bitte reihen Sie die folgenden Themenkreise des GW-Lehrplans für jede einzelne Klasse nach ihrer Wichtigkeit“. Pro Klasse war der für die Lehrperson wichtigste Themenkreis zu identifizieren und mit 1 zu kennzeichnen, der am zweitwichtigsten eingeschätzte mit 2 etc.

Im GW-Lehrplan 2000 existieren zahlreiche Themenkreise, in denen die Vernetzung von geographischen und wirtschaftskundlichen Aspekten deutlich zum Ausdruck gebracht wird. In zwei Themenkreisen werden überwiegend wirtschaftskundliche Aspekte angesprochen, nämlich in „Wirtschaften im privaten Haushalt“ (3. Klasse) und in „Volkswirtschaftliche
Zusammenhänge: Österreich - Europa“ (3. Klasse). Daneben dominieren in folgenden Themenkreisen wirtschaftskundliche Inhalte tendenziell, was eine Analyse der zugeordneten Ziele zeigt: „Gütererzeugung in gewerblichen und industriellen Betrieben" (2. Klasse), „Der Dienstleistungsbereich“ (2. Klasse), „Einblicke in die Arbeitswelt“ (3. Klasse), „Zentren und Peripherien in der Weltwirtschaft" (4. Klasse) und „Leben in der ,Einen Welt" - Globalisierung“ (4. Klasse).

Die beiden Themenkreise „Einblicke in die Arbeitswelt" und „Leben in der ,Einen Welt ${ }^{\star}$ - Globalisierung" sind eher als wirtschaftskundlich dominierte Themenkreise zu identifizieren und werden in ihrer eingeschätzten Bedeutung vor andere Themenkreise des GW-Lehrplans gereiht. Deswegen wird festgehalten, dass eher wirtschaftskundlich geprägten Themenkreisen des GW-Lehrplans 2000 von Lehrpersonen keine generell geringere Bedeutung zugemessen wird. Doch gilt es zu beachten, dass die überwiegend wirtschaftskundlich dominierten Themenkreise "Wirtschaften im privaten Haushalt" und „Volkswirtschaftliche Zusammenhänge: Österreich - Europa" sehr schlechte Werte bei der eingeschätzten Wichtigkeit erzielen. Dies kann auch mit der Ordnung der Themenkreise im Lehrplan 2000 zusammenhängen, weil diese beiden Themenkreise als vorletzter und letzter innerhalb des Schuljahres angeführt werden.

Fazit 8: Da überwiegend wirtschaftskundlich dominierte Themenkreise ("Wirtschaften im privaten Haushalt" und "Volkswirtschaftliche Zusammenhänge: Österreich - Europa") schlechte Bedeutungswerte erzielen, war es bei der Neugestaltung des GW-Lehrplans wichtig, keine erneuten wirtschaftsdominierten Kompetenzbereiche zu schaffen, sondern auf eine möglichst ausgewogene Quantität, Kombination bzw. Integration von geographischen und wirtschaftskundlichen Zielstellungen über alle Kompetenzen hinweg zu achten (vgl. übereinstimmend Zwischenfazit 7). Das bedeutete im Vergleich zum Lehrplan 2000 auch eine Vermeidung von rein geographischen Kompetenzbereichen.

\section{$4 \quad$ Resümee und Ausblick}

Die durch die Vorgängerlehrpläne 1985 und 2000 begründete sozialwissenschaftliche Tradition des Faches wurde im neu gestalteten GW-Lehrplan 2020 durch auch in der sozioökonomischen Bildung und in der GW-Fachdidaktik zentrale Prinzipien wie etwa Lebenswelt-, Subjekt- und Problemorientierung sowie durch sozialwissenschaftliche Multidisziplinarität und Polyparadigmatizität fortgeführt und vertieft. $\mathrm{Zu}$ för- 
dernde Kompetenzen, übergeordnete Leitkompetenzen wie Orientierungs-, Urteils- und Handlungskompetenzen sowie zentrale fachliche Konzepte beziehen sich stets auf das gesamte Wirkungsgefüge Gesellschaft - Politik - Wirtschaft - Umwelt und integrativ auf die lebensweltorientierten Wirtschaftsbereiche Privathaushalt, Konsum, Arbeitswelt und Gesellschaft. Durch die verstärkte Umsetzung der sozioökonomischen Bildung im neuen GW-Lehrplan war es eine richtig einzuschätzende Konsequenz, nicht mehr länger von „Wirtschaftskunde“ im Sinne der Anhäufung und Reproduktion von Wissen zu sprechen, sondern das Fach in "Geographie und Wirtschaftliche Bildung" umzubenennen.

Zum Zeitpunkt des Abschlusses dieses Beitrags, am 05.11.2019, bestehen noch Unabwägbarkeiten bezüglich der Umsetzung des endgültigen Lehrplantexts. Erstens konnte sich der Verfasser immer nur auf ein - bereits sehr weit fortgeschrittenes - Zwischenergebnis des von der ministeriellen Lehrplangruppe formulierten Lehrplantextes beziehen. Es sind weitere Verfeinerungen bei künftigen Treffen geplant, wenngleich auch eine grundlegende Umgestaltung des vorliegenden Entwurfs durch diese Lehrplanarbeitsgruppe wenig wahrscheinlich erscheint. Zweitens werden vonseiten des Bildungsministeriums eventuell noch Änderungswünsche geäußert, die berücksichtigt werden müssen. Drittens wird Österreich zurzeit von einer Übergangsregierung geleitet. Ob eine künftige Bildungsministerin bzw. ein künftiger Bildungsminister den gesamten Lehrplan auf diese Weise verordnen wird, ist aus heutiger Sicht nicht abzuschätzen. Nach dem aktuell (Stichtag 20.02.2020) gültigen ministeriellen Zeitplan soll der Lehrplan im September 2020 kundgemacht und ab dem Schuljahr 2022/23 aufsteigend in der 5. Schulstufe umgesetzt werden.

\section{Literatur}

Anderson, L. W. \& D. R. Krathwohl (Hrsg.) (2001): A Taxonomy for Learning, Teaching, and Assessing. A Revision of Bloom's Taxonomy of Educational Objectives. Longman, New York.

Blättel-Mink, B. \& K.-U. Hellmann (Hrsg.) (2010): Prosumer Revisited: Zur Aktualität einer Debatte. Verlag für Sozialwissenschaften, Wiesbaden.

Bourdieu, P. (1998): Gegenfeuer. Wortmeldungen im Dienste des Widerstands gegen die neoliberale Invasion. UVK, Konstanz.

Bundeskanzleramt (Hg.) (2019): Gesamte Rechtsvorschrift für Lehrpläne - allgemeinbildende höhere Schulen. Eigenverlag, Wien. https://www.ris.bka.gv.at/GeltendeFassung.wxe?Abfrage=Bundesnormen $\&$ Gesetzesnumm er $=10008568(01.11 .2019)$
Bundesministerium für Bildung und Frauen (Hg.) (2012): Die kompetenzorientierte Reifeprüfung Geographie und Wirtschaftskunde. Richtlinien und Beispiele für Themenpool und Prüfungsaufgaben. Wien. https://bildung. bmbwf.gv.at/schulen/unterricht/ba/reifepruefung_ahs_ lfgw_22201.pdf?74wbmy (02.11.2019)

Butterwegge, C., B. Lösch \& R. Ptak (2016): Kritik des Neoliberalismus. 3. Aufl. Springer, Wiesbaden.

Chreiska-Höbinger, C., C. Fridrich, S. Hinsch, P. Hofmann, H. Herbert, M. Vorage, T. Jekel, L. Keller \& A. Koller (2019): Entwurf des Fachlehrplans für den Gegenstand Geographie und Wirtschaftliche Bildung (Stand: 15.11.2019). In: GW-Unterricht 156. S. 74-79.

Crouch, C. (2008): Postdemokratie. Suhrkamp, Berlin.

Engartner, T. \& B. Krisanthan (2014a): Einfallstor Schule. Wie Unternehmen und Stiftungen mit Unterrichtsmaterialien werben. In: WestEnd 11(2). S. 141-153.

Engartner, T. \& B. Krisanthan (2014b): Ökonomische Bildung in Zeiten der Ökonomisierung - oder: Welchen Anforderungen muss sozio-ökonomische Bildung genügen? In: Fischer, A. \& B. Zurstrassen (Hrsg.): Sozioökonomische Bildung. Bonn: Bundeszentrale für politische Bildung. S. 155-176.

Engartner, T. (2019a): Die integrative Kraft sozioökonomischer Bildung - oder: Herausforderungen für die sozialwissenschaftliche Kontextualisierung wirtschaftlicher Phänomene und ökonomischer Logiken. In: GW-Unterricht 153. S. 20-26.

Engartner, T. (2019b): Charakteristika und Perspektiven sozioökonomischer Bildung in Deutschland. In: Österreich Geschichte Literatur Geographie 1 (Industrie - Innovation - Region). S. 84-97.

Engartner, T. (2019c): Wie DAX-Unternehmen Schule machen. Lehr- und Lernmaterial als Türöffner für Lobbyismus. Otto Benner Stiftung, Frankfurt am Main.

Etzioni, A. (1988): The Moral Dimension. Toward a New Economics. The Free Press, New York.

Famulla, G.-E. (2019): Sozioökonomische Bildung Grundgedanken. In: Autorengruppe Sozioökonomische Bildung (Hrsg.): Was ist gute ökonomische Bildung? Leitfaden für den sozioökonomischen Unterricht. Wochenschau Verlag, Frankfurt am Main. S. 19-31.

Fischer, A. \& B. Zurstrassen (2014): Annäherungen an eine sozioökonomische Bildung. In: Fischer, A. \& B. Zurstrassen (Hrsg.): Sozioökonomische Bildung. Bundeszentrale für politische Bildung, Bonn. S. 7-31.

Fridrich, C. (2016): Basiskonzepte in Geographie und Wirtschaftskunde - ein Vorschlag für die Sekundarstufe I. In: Geo Graz 59. S. 24-31.

Fridrich, C. (2019): Von einem engen zu einem integrativen Verständnis - Ein Profil für die ökonomische Bildung im Unterrichtsgegenstand Geographie und Wirtschaftskunde. In: Österreich Geschichte Literatur Geographie 1 (Industrie - Innovation - Region). S. 72-83.

Fridrich., C. \& M. Hofmann-Schneller (2017): Positionspapier „Sozioökonomische Bildung“. In: GW-Unterricht 145. S. 54-57. 
Fridrich, C. \& A. Koller (2019): Zum zweiten Lehrplanentwurf: Zwischenstand, Feedback-Gebende, Perspektiven. In: GW-Unterricht 156. S. 73.

Haarmann, M. P. (2014): Sozioökonomische Bildung ökonomische Bildung unter der Zielperspektive der gesellschaftlichen Mündigkeit. In: Fischer, A. \& B. Zurstrassen (Hrsg.): Sozioökonomische Bildung. Bundeszentrale für politische Bildung, Bonn. S. 206-222.

Hedtke, R. (2015a): Sozioökonomische Bildung als Innovation durch Tradition. In: GW-Unterricht 140. S. 18-38.

Hedtke, R. (Hrsg.) (2015b): Was ist und wozu Sozioökonomie? Springer VS, Wiesbaden.

Hedtke, R. (2018): Das Sozioökonomische Curriculum. Wochenschau Verlag, Frankfurt am Main.

Hedtke, R. (2019): Ökonomisierung: Programm oder Problem? In: bwp@ Berufs- und Wirtschaftspädagogik 35. S. 1-19. http://www.bwpat.de/ausgabe35/hedtke_ bwpat35.pdf (02.11.2019)

Hinsch, S., H. Pichler, T. Jekel, L. Keller \& F. Baier (2014): Semestrierter Lehrplan AHS, Sekundarstufe II. Ergebnis der ministeriellen Arbeitsgruppe. In: GW-Unterricht 136. S. 51-61.

Kaminski, H. (2006): Wie viel Politik braucht die ökonomische Bildung? In: Weißeno, G. (Hg.): Politik und Wirtschaft unterrichten. Schriftenreihe Bd. 483. Bundeszentrale für politische Bildung, Bonn. S. 144-160.

Kaminski, H. (2014): Entwicklung und Institutionalisierung der ökonomischen Bildung als Teil der schulischen Allgemeinbildung. In: Unterricht Wirtschaft + Politik3. S. 50-54.

Klafki, W. (1991): Neue Studien zur Bildungstheorie und Didaktik. Zeitgemäße Allgemeinbildung und kritischkonstruktive Didaktik. 2. Aufl. Beltz, Weinheim/Basel.

Kutscha, G. (2014): Ökonomie an Gymnasien unter dem Anspruch des Bildungsprinzips - Diskursgeschichtlicher Rückblick und Zielperspektiven für die sozio-ökonomische Bildung. In: Fischer, A. \& B. Zurstrassen (Hrsg.): Sozioökonomische Bildung. Bundeszentrale für politische Bildung, Bonn. S. 63-80.

Mikl-Horke, G (Hg.) (2011a): Sozioökonomie: Die Rückkehr der Wirtschaft in die Gesellschaft. Metropolis-Verlag, Marburg.

Mikl-Horke, G. (2011b): Historische Soziologie - Sozioökonomie - Wirtschaftssoziologie. VS Verlag für Sozialwissenschaften, Wiesbaden.
Ötsch, W. O. (2019): Mythos Markt. Mythos Neoklassik. Das Elend des Marktfundamentalismus. Metropolis-Verlag, Marburg.

Pichler H., C. Fridrich, C. Vielhaber \& F. Bergmeister (2017): Der fachdidaktische Grundkonsens 2.0 in der Verbundregion Nordost. Perspektiven einer zukunftsfähigen Orientierungshilfe im GW-Unterricht. In: GW-Unterricht 146. S. 60-62. doi:10.1553/gw-unterricht146s60.

Piorkowsky, M.-B. (2012): Ökonomie des privaten Haushalts. In: May, H. \& C. Wiepcke (Hrsg.): Lexikon der ökonomischen Bildung. 8. Aufl. Oldenbourg Verlag, München. S. 437-440.

Rumpold, H. \& B. Greimel-Fuhrmann (2016): Wirtschaftswissen in der Sekundarstufe I. Entwicklung eines Erhebungsinstruments für die Zielgruppe von Schüler/ inne/n der achten Schulstufe. In: Zeitschrift für ökonomische Bildung 5. S. 119-149.

Schimank, U. \& U. Volkmann (2008): Ökonomisierung der Gesellschaft. In: Maurer, A. (Hg.): Handbuch der Wirtschaftssoziologie. VS Verlag für Sozialwissenschaften. S. 382-393.

Sitte, W. (2001). Wirtschaftserziehung. In: Sitte, W. \& H. Wohlschlägl (Hrsg.): Beiträge zur Didaktik des „Geographie und Wirtschaftskunde“-Unterrichts. Institut für Geographie der Universität Wien, Wien. S. 545-552.

Stieger, S. \& T. Jekel (2019): Ideology, education, and financial literacy. Uncovering neoliberal ideology in assessment studies of economics education - the case of Austria. In: Journal of Social Science Education 18(2). S. 4-26. doi:10.4119/jsse-906

Tenfelde, W. \& T. Schlömer (2012): Ökonomische Bildung. In: May, H. \& C. Wiepcke (Hrsg.): Lexikon der ökonomischen Bildung. 8. Aufl. Oldenbourg, München. S. $440-443$.

Thiele-Wittig, M. (2003): Kompetent im Alltag: Bildung für Haushalt und Familie. http://www.bpb.de/ apuz/27769/kompetent-im-alltag-bildung-fuer-haushaltund-familie? $\mathrm{p}=$ all $(03.11 .2019)$

Uhlenwinkel, A. (2018): Ergebnisorientiert und interessensgeleitet - Studien der Wirtschaftslobby zum GWUnterricht. In: GW-Unterricht 150. S. 20-33. https:// www.austriaca.at/0xc1 aa5576\%200x0039127c.pdf (02.11.2019) 